\title{
A psychological intervention for suicide applied to non-affective psychosis: the CARMS (Cognitive AppRoaches to coMbatting Suicidality) randomised controlled trial protocol
}

Patricia A. Gooding ${ }^{1,2^{*}}$ (D), Daniel Pratt ${ }^{1,2}$, Yvonne Awenat ${ }^{1,2}$, Richard Drake ${ }^{1,2}$, Rachel Elliott ${ }^{3}$, Richard Emsley ${ }^{4}$, Charlotte Huggett ${ }^{1,2}$, Steven Jones ${ }^{5,6}$, Navneet Kapur ${ }^{1,2}$, Fiona Lobban ${ }^{5,6}$, Sarah Peters ${ }^{1}$ and Gillian Haddock ${ }^{1,2}$

\begin{abstract}
Background: Suicide is a leading cause of death globally. Suicide deaths are elevated in those experiencing severe mental health problems, including schizophrenia. Psychological talking therapies are a potentially effective means of alleviating suicidal thoughts, plans, and attempts. However, talking therapies need to i) focus on suicidal experiences directly and explicitly, and ii) be based on testable psychological mechanisms. The Cognitive AppRoaches to coMbatting Suicidality (CARMS) project is a Randomised Controlled Trial (RCT) which aims to investigate both the efficacy and the underlying mechanisms of a psychological talking therapy for people who have been recently suicidal and have non-affective psychosis.

Methods: The CARMS trial is a two-armed single-blind RCT comparing a psychological talking therapy (Cognitive Behavioural Suicide Prevention for psychosis [CBSPp]) plus Treatment As Usual (TAU) with TAU alone. There are primary and secondary suicidality outcome variables, plus mechanistic, clinical, and health economic outcomes measured over time. The primary outcome is a measure of suicidal ideation at 6 months after baseline. The target sample size is 250 , with approximately 125 randomised to each arm of the trial, and an assumption of up to $25 \%$ attrition. Hence, the overall recruitment target is up to 333. An intention to treat analysis will be used with primary stratification based on National Health Service (NHS) recruitment site and antidepressant prescription medication. Recruitment will be from NHS mental health services in the North West of England, UK. Participants must be 18 or over; be under the care of mental health services; have mental health problems which meet ICD-10 non-affective psychosis criteria; and have experienced selfreported suicidal thoughts, plans, and/or attempts in the 3 months prior to recruitment. Nested qualitative work will investigate the pathways to suicidality, experiences of the therapy, and identify potential implementation challenges (Continued on next page)
\end{abstract}

\footnotetext{
*Correspondence: patricia.gooding@manchester.ac.uk

1 Division of Psychology and Mental Health, School of Health Sciences,

Manchester Academic Health Sciences Centre, University of Manchester,

Coupland Building 1, Oxford Road, Manchester M13 9PL, UK

${ }^{2}$ Greater Manchester Mental Health NHS Trust (formerly Manchester Mental

Health and Social Care Trust), Manchester, UK

Full list of author information is available at the end of the article
}

C C The Author(s). 2020 Open Access This article is licensed under a Creative Commons Attribution 4.0 International License, which permits use, sharing, adaptation, distribution and reproduction in any medium or format, as long as you give appropriate credit to the original author(s) and the source, provide a link to the Creative Commons licence, and indicate if changes were made. The images or other third party material in this article are included in the article's Creative Commons licence, unless indicated otherwise in a credit line to the material. If material is not included in the article's Creative Commons licence and your intended use is not permitted by statutory regulation or exceeds the permitted use, you will need to obtain permission directly from the copyright holder. To view a copy of this licence, visit http://creativecommons.org/licenses/by/4.0/ The Creative Commons Public Domain Dedication waiver (http://creativecommons.org/publicdomain/zero/1.0/) applies to the data made available in this article, unless otherwise stated in a credit line to the data. 
(Continued from previous page)
beyond a trial setting as perceived by numerous stake-holders.

Discussion: This trial has important implications for countering suicidal experiences for people with psychosis. It will provide definitive evidence about the efficacy of the CBSPp therapy; the psychological mechanisms which lead to suicidal experiences; and provide an understanding of what is required to implement the intervention into services should it be efficacious.

Trial registration: ClinicalTrials.gov (NCT03114917), 14th April 2017. ISRCTN (reference ISRCTN17776666 https://doi.org/10. 1186/ISRCTN17776666); 5th June 2017). Registration was recorded prior to participant recruitment commencing.

Keywords: Suicide, Suicidal thoughts and behaviours, Psychological interventions, Cognitive therapy, Psychosis, Schizophrenia, Psychological suicide mechanisms, Randomised controlled trial

\section{Background}

Suicidal thoughts, plans, and fatalities are of substantial concern worldwide. Approximately 800,000 lives are lost to suicide, globally, every year, which equates to one person dying by suicide every $40 \mathrm{~s}$ [1]. Estimates indicate that approximately 48,344 people died by suicide in the USA in 2018, which is 14.2 deaths per 100,000 population [2]. In the UK, for 2018 registrations, there were 6507 suicide fatalities, that is, 11.2 deaths per 100,000 population [3]. Suicide deaths increased in 2018 in the UK compared to 2013 figures [4]. In the US, the suicide death rate has also been increasing over time [5]. Suicide attempts are far more prevalent than suicide deaths, and suicide thoughts are more frequent than attempts. For example, as collected by the Adult Psychiatric Morbidity Survey, in 2014, 21.6\% of British white people in England, UK, had suicidal thoughts whereas $6.9 \%$ had attempted suicide [6], and $0.01 \%$ had died by suicide that year. There were 1.4 million suicide attempts in 2017 in the USA [2] and 47,000 suicide deaths [7].

There is sound evidence that suicidal thoughts, plans, attempts and deaths are significantly elevated in those with severe mental health problems [8]. For example, mental health problems in those experiencing nonaffective psychoses, or recent onset psychosis, have been associated with one of the highest, relatively unchanging, suicide mortality rates across the decades [9-12]. Whilst it is vital to focus on preventing deaths by suicide it is equally important to focus on reducing suicidal thoughts, plans and acts in people with severe mental health problems because they are both common and highly distressing for individuals and their families. For example, up to $50 \%$ of people with non-affective psychoses will experience suicidal thoughts and/or suicide attempts [10, 13]. Furthermore, suicidal thoughts and acts are associated with immense psychological distress [14] and can be strong predictors of suicide fatalities [15]. For instance, as reported by a recent meta-analysis, people with schizophrenia who also had thoughts of suicide were six times more likely to die by suicide compared to a 1.5 fold increase in those experiencing depression [16]. Hence, it is important that interventions which combat suicidal thoughts and acts are designed to attenuate a range of suicidal experiences, such as thoughts, urges, plans and attempts, in people with severe mental health problems such as psychosis.

A small number of meta-analytic systematic reviews have examined the extent to which psychological and psychosocial interventions reduce self-harm and suicidal experiences in those with mental health problems [17-21]. For example, two reported that psychological interventions were effective as long as they targeted suicidality (i.e., suicidal thoughts, behaviours and acts) or psychological precursors of suicidal experiences (e.g., hopelessness) rather than symptoms reflecting a psychiatric diagnosis $[18,19]$. One of these reviews specifically evaluated the effects of cognitive-behavioural types of therapies (CBT) on suicide thoughts and behaviours [19]. Findings showed that cognitive behavioural therapies, including dialectical behavioural therapy, reduced suicidal thoughts and behaviours.

An issue with many studies included in such reviews is that few tested the effectiveness of a psychological talking therapy which, first of all explicitly focused on suicidal thoughts and acts, and second, were based on empirically supported recent psychological models which attempt to delineate the psychological pathways underpinning suicidal thoughts and behaviours. The Schematic Appraisal Model of Suicide (SAMS) [22] is one such recent psychological model of suicide which was developed from the influential Cry of Pain model [23] but also resonates with other contemporary psychological models of suicide, such as the Integrated Motivational Volitional Model [24-26]. These contemporary models converge, at least to some extent, in highlighting the importance of perceptions of emotional dysregulation, lack of social support and a perceived inability to solve inter-personal problems together with experiences of feeling defeated, trapped and hopelessness in pathways to suicidal thoughts and acts. It is important to understand mechanisms underlying suicidal experiences which are transdiagnostic and appear to be activated or 
triggered regardless of specific mental health problems or psychiatric diagnoses but to also recognise that specific symptoms associated with mental health problems, such as hallucinations, delusions, and hyperarousal may amplify suicidal thoughts, plans and acts [27-29].

Psychological therapies are optimally efficacious and effective if they focus on evidence-based, theoretically derived, psychological mechanisms thought to underlie suicidal thoughts and behaviours, and if as part of the therapy, they address such suicidal experiences explicitly [19]. Some of the cognitive behavioural therapies which have been evaluated may not always have been explicitly focused in this way. One psychological intervention approach which has focussed on ameliorating suicidal thoughts and acts is Cognitive Behavioural Suicide Prevention (CBSP) $[14,19,30]$. CBSP is based on the SAMS [22]. It was developed by the current authors and colleagues $[14,30,31]$ as a psychological intervention designed to target suicidal thoughts, urges, plans and acts by focusing on perceptions of poor emotional regulation, social isolation, and difficulties with interpersonal problem solving. As these negative appraisals are posited to give rise to perceptions of being defeated, trapped and hopeless over time [32], and, in accord with a large body of evidence showing that these perceptions are central in the pathways to suicidal experiences [28, 32-42], CBSP also targets perceptions of defeat, entrapment and hopelessness. Hence, CBSP is unique in i. being grounded in a testable, evidence based psychological model of suicidal thoughts and acts, and ii. having an explicit and central focus on suicidal thoughts and behaviours.

Three pilot Randomised Controlled Trials (RCTs) have shown that the CBSP intervention is feasible and acceptable when adapted for use across a range of settings, and have suggested that it can be efficacious in reducing suicidal thoughts and acts. The first found reductions in measures of suicidal ideation and suicidal probability in people experiencing non-affective psychosis who lived in the community $[30,43]$. The second, found that suicidal behaviours were less frequent in male prisoners following the CBSP intervention [31]. The third, found that this intervention was feasible, acceptable, and safe, and suggested that it may be cost-effective when used in psychiatric in-patient settings [44]. This means that the evidence from pilot RCTs across three diverse settings indicated that the CBSP suicide focussed therapy has the potential to counter suicidal experiences.

In order to develop this work further it is important to test the efficacy of CBSP in a larger, definitive, RCT in people with severe mental health problems who are vulnerable to suicidal experiences. It is also important to test the extent to which the underlying psychological mechanisms, on which CBSP is based are supported when applied to people with non-affective psychosis.
Therefore, we propose to test CBSP adapted for people with non-affective psychosis (CBSPp) in an RCT, with two arms $i$. the CBSPp intervention plus treatment as usual (CBSPp plus TAU) and ii. treatment as usual (TAU) across three time points of baseline, 6 months follow-up (at therapy cessation), and 12 months followup. Our CARMS RCT is novel because i. it is founded on a testable psychological theory, ii. it is based on pilot work in diverse settings of a suicide focused cognitive therapy, and iii. unlike many of the studies included in recent meta-analytic reviews [17-21], it attempts to understand the mechanisms and the efficaciousness of a psychological 'talking' therapy in a population of individuals with severe mental health problems who are highly vulnerable to suicidal thoughts and acts. A more complex trial design is inappropriate given that the CARMS RCT advances pilot work.

The primary outcome is suicidal thoughts and acts at 6 months following entry to the trial. It is predicted that i. suicidal thoughts and acts will be less frequent and less severe in the intervention condition compared to the control condition, measured at therapy cessation (6 months) and after a 12 month follow up (FU) period compared to baseline; ii. negative appraisals of social support, emotional regulation, and interpersonal problem solving will lead to stronger perceptions of being defeated, trapped and hopeless, which will in turn lead to suicidal thoughts and behaviours over time; and iii. the treatment condition will result in less severe negative appraisals, and reduced perceptions of defeat, entrapment and hopelessness compared to the control condition. A more exploratory prediction is that symptoms of psychosis, particularly positive symptoms of hallucinations and delusions and their associated distress, will strengthen the relationships between negative appraisals, perceptions of defeat, entrapment and hopelessness and suicidal thoughts and behaviours. The acceptability and cost-effectiveness of the intervention will also be examined. Regarding the CBSPp therapy, we will attempt to gain information about the therapy process, the therapeutic alliance, engagement and adherence.

\section{Methods/Design}

\section{Patient and public involvement (PPI)}

A central, and fundamentally important component of the CARMS RCT is that people who are Experts-ByExperience (EBEs) have been involved in the design and set up of this project. Indeed, some of the coinvestigators on the CARMS trial have personal experience of suicidality. We have established a CARMS specific PPI group with suicidal experiences and severe mental health problems who will be invited to have direct involvement in all the research stages, decision making processes, and suicidality training processes, of the 
CARMS project throughout its duration. It is becoming increasingly recognized that this type of PPI involvement is essential in any research projects investigating mental and physical health issues including those with economic implications [45-48].

\section{Study design: the RCT}

The CARMS trial involves both an RCT and a nested qualitative component within the design. The trial was registered with ClinicalTrials.gov (reference NCT0311491 7) where it was first posted 14th April 2017. It was also registered with ISRCTN (reference ISRCTN17776666 https://doi.org/10.1186/ISRCTN17776666) with registration being assigned 5th June 2017). Registration was accepted before recruitment commenced. The design of the trial follows Consolidated Standards of Reporting Trials (CONSORT; http://www.equator-network.org/reportingguidelines/consort/) and SPIRIT guidelines (Standard Protocol Items: Recommendations for Intervention Trials; http://www.spirit-statement.org/), and the TIDieR checklist and guide; Template for Intervention Description and Replication (BMJ 2014; 348:g1687).

The design is a single-blind RCT with two parallel arms. Outcome variables assessing suicidal experiences will be collected at baseline, and at 6 and 12 months follow-up time points. Data pertaining to clinical and mediational variables will be collected at baseline, after therapy cessation (6 months), and at the 12 month follow-up time point. Health economics measures (assessed over the previous 6 months) will be collected at baseline and at the 12 month follow-up time-point to allow comparison over the pre-trial and post randomization period. It is anticipated that up to 24 individual therapy sessions, of up to an hour, will be offered over 6 months. Participants will be independently randomised to one of the two trial arms, with stratification based on whether the participant is or is not prescribed anti-depressant medication (as this could affect the primary outcome variable) and the NHS sites over which the trial is being tested (see Fig. 1).

\section{Study design: the qualitative component}

There are four qualitative work-streams. The aim of the first is to investigate perceptions of both participants and health professionals, including mental health professionals, concerning aspects of the implementation of a psychological talking therapy focused on ameliorating suicidal thoughts and behaviours in people experiencing non-affective psychosis. The second work-stream explores psychological mechanisms which are perceived to be precursors and drivers to suicidal thoughts and acts. The third, examines acceptability of the experience of the therapy from the perspective of those randomised to
CBSPp. The fourth work-stream investigates the experiences of participants taking part in research which has a suicide focus.

A purposive sampling matrix will be used to maximise diversity in the participants recruited to the qualitative work streams. Semi-structured, one-to-one, interviews will be used across all the qualitative work streams to generate the data.

\section{Recruitment to the RCT (the host site is Greater Manchester Mental Health NHS Foundation Trust)}

Potential participants will be identified by mental health professionals in the participant's mental health care team (including community mental health teams, early intervention services, and inpatient psychiatric wards) using the inclusion and exclusion criteria listed below. These professionals will then ascertain whether participants are willing to receive information about the study and to be contacted by a CARMS researcher. If so, potential participants will be contacted in order to provide them with a Participant Information Sheet (PIS) and to check eligibility for the trial. At least $24 \mathrm{~h}$ after receiving the PIS, participants will be asked to confirm whether they want to take part in the CARMS project and, if so, to provide informed consent.

Posters and flyers will be distributed in areas accessible to potential participants e.g., health service waiting rooms, inpatient wards, relevant community venues, where feasible. In addition, information about the CARMS trial will be disseminated via mental health charities. Recruitment materials will be used to enable participants to request that a member of their mental health care team (e.g., care co-ordinator) refer them into the CARMS trial. A dedicated CARMS website will also be used to provide information about the CARMS trial (https://sites.manchester.ac.uk/carms/). In addition, recruitment materials (e.g., posters, flyers, newsletters) will be distributed to mental health service providers. Members of the CARMS team, including researchers and therapists, will deliver mental health team briefings about CARMS as appropriate.

\section{Study design: eligibility criteria}

The eligibility criteria are i. ICD-10 diagnosis relating to non-affective psychosis (ICD 10, i.e., F20 - F29), ii. selfreported experiences of suicidal thoughts, urges, plans and/or acts in the past 3 months, iii. in contact with mental health services and under the care of a mental health services clinical team (e.g., NHS community or inpatient mental health care teams, early intervention teams, crisis teams, home treatment teams), iv. aged 18 or over, v. sufficient English to complete questionnaires (hence, not needing an interpreter), vi. able to give informed consent as assessed by either a responsible 


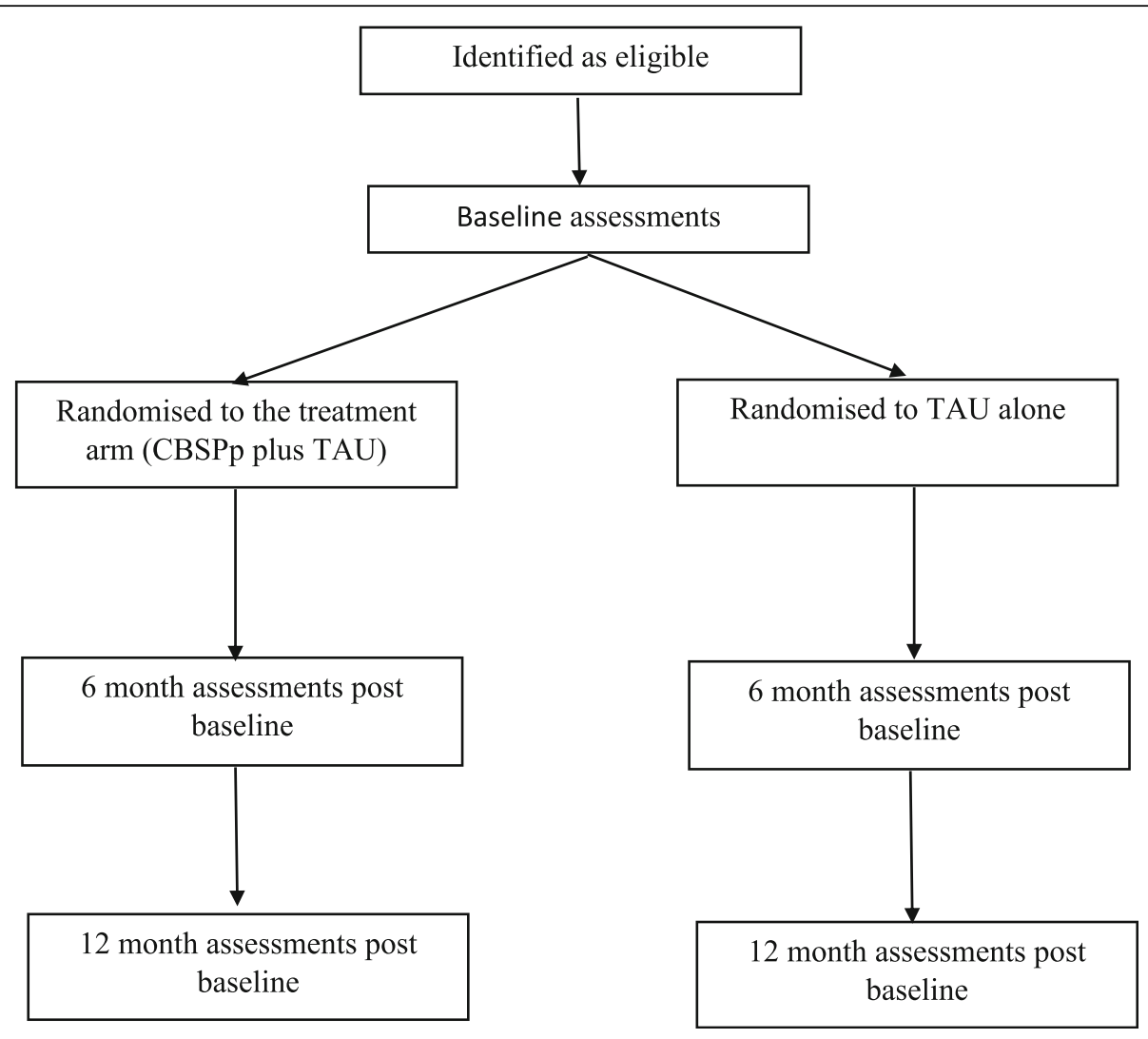

Fig. 1 The CARMS Trial design

clinician or by CARMS research staff following the British Psychological Society's guidelines on gaining informed consent (http://www.bps.org.uk/sites/default/ files/documents/code_of_human_research_ethics.pdf).

The exclusion criteria are i. dementia, or an organic brain disorder, ii. unable to complete assessments due to language barriers, and iii. currently taking part in a clinical trial. A short screening procedure will be used to assess eligibility.

\section{Study design: withdrawal criteria}

The withdrawal criteria are i. the participant decides to withdraw from the trial for any or no reason, and ii. the participant is lost to follow-up. However, data will be included in the analyses up to the point of withdrawal with appropriate consent.

\section{Study design: randomisation and blinding}

Randomisation will take place only when participants have consented to participate and after the baseline assessments have been completed. Hence, participants will consent to take part without knowing their group allocation. The Manchester Academic Health Sciences Centre (MAHSC), Clinical Trials Unit (CTU) will oversee the blocked stratified randomisation procedures. Researchers conducting the assessments, the trial statistician, one of the CARMS Trial Co-Principal Investigators, and members of the CTU conducting data handling and data monitoring will be blind to the randomised allocation group of participants. Procedures will be in place if a CARMS researcher becomes unblinded. In such an instance of unblinding then a different researcher will be allocated to work with that participant. We will not disclose the reason for this allocation. Indeed, we will strategically re-allocate researcher resource so that reallocation is a frequent occurrence for researchers, especially given the wide geographical distribution of sites.

\section{Study design: sample size and power calculations}

Based on power analyses, the target sample size is 125 participants in each arm (treatment and control), meaning that there will be a target of 250 participants in total for the primary analyses at 6 months across the participating sites. To account for attrition of $25 \%$, it is anticipated that up to 333 participants will be recruited into the baseline phase of the trial. It is estimated that $50 \%$ of people approached will decline to take part. Therefore, approximately 666 participants will be identified as eligible and screened. 
We will use an approach based on a simple t-test for the between group comparison in the primary suicide outcome measure which is specifically designed to account for differential clustering or partial nesting between the two arms [49]. It is implemented using the command clsampsi- in Stata [50]. This approach requires the following assumptions:

a. Effect size: A clinically meaningful difference on the primary suicide outcome variable (ASIQ) from baseline to the 6 month follow-up time-point is estimated as a 16 point reduction, which corresponds to an effect size of 0.42 , based on pilot data [30].

b. Attrition: We are allowing for an attrition rate of $25 \%$ from baseline to final follow-up.

c. Clustering: we account for clustering of outcomes within the participants sharing a therapist in the treatment arm with an ICC $=0.02$.

d. Random allocation: 1:1 random allocation, 0.05 significance level, and $80 \%$ statistical power.

For our proposed mediational analyses to test psychological mechanisms, a sample size of approximately 250 has greater than $80 \%$ power to detect a proportion mediated of $35 \%$, and over $70 \%$ power to detect a proportion mediated of $30 \%$ (calculated using PowerMediation in R).

In sum, our sample size will have sufficient power for our proposed efficacy and mediation analyses.

\section{Study design: study arms of treatment and control groups}

Treatment arm: the CBSPp intervention plus TAU anticipated as 24 weekly 50 min sessions

The CBSPp intervention is a one-to-one, formulation based approach. Initial sessions focus on engaging the individual in exploring their suicidal thinking and behaviours and how they relate to key underlying psychological constructs and to their key emotions, cognitions and behaviours. Ways in which these types of thoughts and behaviours interact with their experiences of psychosis, life circumstances and history is also explored to reach a shared formulation and understanding of their suicidal thoughts, plans, and acts. The approach, collaboratively, generates possible actions which are likely to modify negative appraisals of emotional regulation, improve perceptions of social support, and perceptions of interpersonal problem solving, and to influence other main drivers of their suicidal thoughts and acts, for example, distressing symptoms of psychosis. As a consequence, it is hypothesised that perceptions of defeat, entrapment, and hopelessness will be improved indirectly, although, perceptions of defeat, entrapment and hopelessness will also be worked on directly during the therapy [14, 30, 31]. Therapists will be trained in CBT techniques and further trained in CBSPp.

\section{Control arm: TAU only}

TAU will include usual clinical care. This is likely to be, although not exclusively, in the form of assessment and intervention from a multi-disciplinary mental health team as an outpatient or inpatient, prescription of psychiatric medication, regular monitoring, and social support. Where possible, we will document the types of TAU offered to and/or received by participants.

\section{Proposed outcome measures: primary, secondary, mechanistic, clinical, therapy process, and health economics variables}

Primary suicide outcome measure This is the Adult Suicidal Ideation Questionnaire [ASIQ] [51] which is a self-report measure comprising 25 items. Suicide ideation is a predictor of suicide attempts and suicide death [52]. Participants are asked to report the frequency of thoughts about death and suicide in the last month using a 7 point Likert scale. For individuals attending psychiatric outpatient clinics with a history of suicide attempts, internal consistency was reported as .97. Testretest reliability was also reported to be high $(r=.95)$ in a mixed sample including those with a history of suicide attempts [51]. The primary analyses examine changes in the ASIQ from baseline to the 6 month time-point.

Secondary suicide outcome measures We will use the following measures, the first two of which are self-report measures.

1. The Suicide Probability Scale [53] comprises 36 items and measures four components of suicidal experiences including suicidal ideation, hopelessness, negative selfevaluations and hostility. The internal reliability for this measure was found to be high in a sample including psychiatric inpatients with a Cronbach's alpha score reported as .93 [53].

2. The Beck Scale for Suicidal ideation [54] measures recent suicidal ideation, plans, and intent over the past week (19 items) together with previous attempt history (2 items). This self-report measure has been reported as having an alpha coefficient of 0.96 and test-retest reliability of $\mathrm{r}=0.88$ with people who were psychiatric inpatients and with those with experiences of non-affective psychosis [55].

3. Self-reported frequency of suicidal thoughts, plans and attempts over the past 6 months. Where feasible, we will collect data from clinical case notes and documentation of adverse events, including those considered to be serious. 
Mechanistic outcome variables Each mechanistic variable assesses key components of the predicted psychological pathways to suicidal experiences [22] using selfreport questionnaires:

1. The Difficulties in Emotional Regulation Scale [56] measures self-reported appraisals of emotional control. Four aspects of emotional regulation comprise the scale which are i. an awareness and reflective capacity, or understanding, with respect to emotions, ii. the ability to accept thoughts and behaviours reflective of emotions, iii. feeling in control of emotional reactions, and iv. feeling able to use effective emotional regulation strategies. Typically, total scores on this scale have been found to correlate positively with a range of measures of psychological mental health problems in a large sample presenting at an outpatient psychiatric clinic. In addition, internal reliability was reported as 0.97 in this sample [57].

2. The Social Problem-Solving Inventory Short Form [58] tests appraisals of social problem solving and has five sub-scales (positive problem orientation, negative problem orientation, rational, impulsive, and avoidant problem solving styles). Overall, there are 25 items. Alpha reliabilities have been reported as ranging between 0.85 and 0.96 in adolescents, young adults, middle aged and elderly adults [58].

3. The Social Support Appraisals Scale [59] assesses perceptions of available social support using 23 items. Data generated from five heterogeneous student/college samples and five community samples indicated Cronbach's alpha reliability calculations to be between 0.80 and 0.90 [59].

4. The Beck Hopelessness Scale [60] measures perceptions of having a negative future with 20 , binary choice, yes/no responses. Hopelessness has been shown to be a robust predictor of suicidal experiences even compared with measures of depressed mood states [61]. Reliability estimates have been recorded as exceeding 0.88 in a range of samples including those with suicidal thoughts and behaviours and severe mental health problems $[60$, 62, 63].

5. The Defeat and Entrapment scales [64] assess perceptions of being defeated and trapped both of which use 16 items. Perceptions of both defeat and entrapment have been robustly implicated in pathways to suicidal experiences in people experiencing depression, anxiety [32], and also in those with non-affective psychosis [28]. In people with non-affective psychosis who also experienced some level of suicidal thoughts and behaviours, in the past or currently, the Cronbach's alpha coefficients were reported to be 0.86 for defeat and 0.95 for entrapment [28].

Clinical variables Mental health problems pertaining to psychosis, depression, and functioning, are measured in clinical interviews as follows: Positive and Negative Syndrome Scale [PANSS] [65], the Psychotic Symptom Rating Scales [PSYRATS] [66], the Personal and Social Performance Scale [PSP] [67] and the Calgary Depression Scale [CDS] [68]. The PANSS is the most widely used measure of positive, negative and general symptoms experienced by people with psychosis and was shown to have sound psychometric properties using a response analysis [69]. The PSYRATS shows excellent reliability across raters and has robust validity [66]. The PSP has excellent face validity and reliability with the majority of ratings taking place in those with schizophrenia [67]. The CDS was specifically designed for people with schizophrenia and was reported as having excellent reliability estimates [68].

Information about current medication for mental health problems (e.g., anti-psychotic and anti-depressant medication) will be recorded from self-reports and supplemented from clinical records where possible.

Health economics measures Participant-level costs will be generated for each participant in both the CBSPp treatment and TAU control arms. Costs will comprise the costs of the intervention, of TAU, and downstream costs. Costs will be constructed from a combination of trial-based resource use (NHS contacts and the Client Service Use Receipt Inventory [CSRI] amended for our trial, to monitor service use) with published unit costs, allowing comparison of CBSPp with TAU in terms of costs to the NHS and PSS. Several methods are available to collect resource-use. The most popular have been resource-use questionnaires, resource-use diaries, and electronic record searches [70-72]. The Clients Service Receipt Inventory (CSRI) [73] is a commonly used method for the retrospective collection of resource-use information which has been implemented in the economic evaluation of a variety of physical and mental health care interventions [74-77]. The CSRI covers a range of economic factors including participant's use of health and social care services, accommodation and living situation, income, employment and benefits (the CSRI is available from the Database of Instruments for Resource-use Measurement [DIRUM] website: http:// www.dirum.org/assets/downloads/634462388066137028CSRI.pdf). Similarly to other studies, we are using a modified version of the CSRI, based on the format and questions of the CSRI but 'modified' to reflect the care pathways of our study participants. Examples include the 
use of mental health care services (community teams, crisis teams, inpatient psychiatric admission),

The EQ-5D-3 L can reflect different levels of psychosis symptomatology, be responsive to intervention effects [78-80], and correlates with the PANSS and Brief Psychiatric Rating Scale - Expanded (BPRS-E) [81]. However, the EQ-5D-3 L showed significant ceiling effects compared with PANSS and BPRS-E. The EQ-5D-5 L [82] is less prone to ceiling effects in a range of health problems [8386] and the increased sensitivity of EQ-5D-5 L may also favour Quality-Adjusted Life-Years (QALY) gains even if the changes in utility are smaller [87]. Hence we will use will use the EQ-5D-5 L. QALYs will be calculated by attaching available utility weights to the health states generated from the EQ-5D-5 L, using area under the curve methods with an assumption of a linear change between time points, controlling for baseline.

Demographic information We will collect demographic information at baseline and then check at follow-up whether factors such as relationship status, work status and education have changed at the two follow-up periods of 6 and 12 months. We will collect data concerning age, ethnicity, gender, type of work, length of time doing this work, and highest level of education.

Assessment of mood A visual analogue scale (0-100) will be used to assess mood prior to all assessment sessions, and after those sessions where possible. This is to get a very quick rating of whether the sessions have negatively impacted mood and will be used to feed into assessments of suicide risk.

Therapy process measures of therapeutic alliance, engagement and adherence For those in the therapy arm of the trial, the therapeutic alliance will be assessed twice, after approximately four sessions and towards the end of the therapy, with the Working Alliance Inventory - short form [88] completed by the therapist and the participant. Therapists will also record the following information for each participant in the therapy arm of the trial as appropriate and where possible: $i$. number of sessions attended and ii. duration of each session.

\section{Data analyses of the CARMS RCT}

\section{Analysis of the trial data to assess efficacy}

A statistical analysis plan will be prepared before any analysis is undertaken, and agreed with the independent DMEC and TSC. Reporting will follow the CONSORT for Psychological and Social Interventions. All analyses and summary statistics will be conducted on the Intention-To-Treat (ITT) population which is defined as all participants randomised regardless of completion of therapy or withdrawal from the study. All analyses will be carried out at the end of the last follow-up assessments, and no interim analysis is planned.

Consideration will be given to potential biases arising from loss to follow-up.

Analyses will be conducted in Stata version 16 or later. Descriptive statistics within each randomised group will be presented for baseline values. These will include counts and percentages for binary and categorical variables, and means and standard deviations, or medians with lower and upper quartiles, for continuous variables, along with minimum and maximum values and counts of missing values. There will be no tests of statistical significance or confidence intervals for differences between randomised groups on any baseline variable.

Treatment effects on primary and secondary outcomes will be estimated using linear mixed models fitted to outcome variables at all time points. Fixed effects will be NHS site, anti-depressant use at baseline (yes/no), baseline assessment for the outcome under investigation, treatment, time and time*treatment interactions. Participant will be included as a random intercept to account for repeated measures. If the number of therapists is different from the number of NHS sites, we will include therapist as an additional random effect.

Marginal treatment effects will be estimated for primary outcome (ASIQ score at 6 months), and for primary and secondary outcomes at all other time points, and reported separately as adjusted mean differences in scores between the groups with $95 \%$ confidence intervals and 2-sided $p$-values. Cohen's D effect sizes will be calculated as the adjusted mean difference of the outcome divided by the sample standard deviation of the outcome at baseline. These will be displayed in a forest plot showing the treatment effects on the primary and the secondary outcomes at 6 months.

We will allow for the presence of missing data under the assumption that the data are Missing At Random, conditional on the covariates in the model, and as a sensitivity analysis include other baseline measures as predictors of future loss to follow-up.

To account for the possible prognostic effect of antidepressant medication on outcomes, we will include anti-depressant use at baseline (yes/no) as a stratifying factor. We acknowledge that use of anti-depressant medication after randomisation might account for a proportion of any observed treatment effect because it might lie on the causal pathway between randomisation and outcome but it is not targeted by the intervention itself. If there is a significant differential effect in the uptake of anti-depressant medication between the treatment and control groups, we will assess the role of antidepressant medication as a mediator, in addition to our hypothesised target mediators. 


\section{Analysis of the trial data to assess mechanisms}

We will perform this analysis using methods similar to those of Baron and Kenny [89] but advanced by newer approaches of structural equation models, instrumental variable analyses, and principal stratification to allow for hidden confounder variables [90-92]. Moderator analyses will focus on examining the effects of psychotic symptoms as an amplifier [93]. Stata version 16 or later will be used for all the analyses.

\section{Analyses of the qualitative work streams}

It is anticipated that data will be largely analysed using an inductive Thematic Analysis (TA) [94, 95] approach taking an interpretative stance. Coding will be undertaken inductively at the manifest level. Following familiarisation, a coding framework will be developed and codes assigned to themes. Data generation and analysis will occur in parallel using a constant comparative approach [96]. Disconfirming evidence will be sought and the analysis refined accordingly where possible. Data generation will cease when theoretical saturation appears to have been achieved. Regular discussion of emerging codes and themes will take place with the wider research team which includes service users, clinical and academic psychologists, and psychiatrists. This is a recognised method for maximising the trustworthiness of the final analysis [97].

\section{Economic evaluation}

Patient-level costs will be generated for each participant in the treatment (CBSPp intervention plus TAU) and TAU alone arms of the trial. Costs will comprise those of the treatment arm, those of TAU arm, and downstream costs. Costs will be constructed from a combination of trial-based resource use (NHS contacts and the Client Service Use Receipt Inventory [CSRI] amended for our proposed trial, to monitor service use) with published unit costs, allowing comparison of treatment with TAU in terms of costs to the NHS and PSS. The CSRI [98] is a commonly used method for the retrospective collection of resource-use information which has been implemented in the economic evaluation of a variety of physical and mental health care interventions [75, 76, 99, 100]. The CSRI covers a range of economic factors including a participant's use of health and social care services, accommodation and living situation, income, employment and benefits (CSRI is available from the Database of Instruments for Resource-use Measurement (DIRUM) website: (http://www.dirum.org/assets/downloads/634462388066137028-CSRI.pdf). Similarly to other studies, we are using a modified version of the CSRI, based on the format and questions of the CSRI but 'modified' to reflect the care pathway of our study participants.
Participant-level costs will be generated for each participant in the Treatment and TAU arms from a combination of trial-based resource use with published unit costs. The unit costs of resource use will be taken from publicly available sources including current editions of NHS reference costs and the Unit Costs of Health \& Social Care [101, 102]. Costs will be compared between the two groups using a bootstrapped regression model (as the data are likely to be skewed).

\section{Custodians of the data}

It should be noted that the Co-Principal Investigators are the custodians of the data.

\section{Discussion}

The risk of suicide fatalities in people who experience severe mental health problems, including those with non-affective psychosis, is extensively elevated compared to suicide fatalities in the general population $[8,10,103]$. In addition, the distress of experiencing suicidal thoughts and acts can be severe, perhaps, especially when linked with mental health problems, such as, hallucinations and delusions $[9,104-106]$. Within the existing literature, there has been a tendency to focus on the extent to which therapies, including psychological talking therapies, result in the reduction of symptoms indicative of mental health problems rather than on redressing suicidal experiences [14, 30, 31, 107]. Evidence indicates that suicidal experiences need to be targeted by psychological talking therapies directly $[18,30$, $31,108]$. However, the development of talking therapies for suicidal experiences is in its infancy [14, 30, 31, 107, 109]. The CARMS project, i.e., a multifaceted RCT, is important because it redresses this gap.

Six strengths of the CARMS RCT should be emphasised, and discussed.

First, the therapy being evaluated in the CARMS RCT is based on a theoretical psychological model, namely the SAMS, of the psychological pathways thought to underlie suicidal experiences [22]. The SAMS resonates with other recent psychological approaches to understanding suicidal thoughts and acts [23-26]. Both the SAMS, and contemporary psychological models of suicidal experiences, have gained support from qualitative, quantitative and epidemiological methods [22, 28, 3234, 63, 110-119].

Second, the CARMS CBSPp intervention has been developed from evidence based on working with people with a range of severe mental health problems, such as those given diagnoses of schizophrenia, PTSD, the bipolar disorders and depression [32, 62, 63, 107, 111-115, 120]. This means that the intervention has a mechanistic, scientific basis which examines both transdiagnostic and the specificities of mental health problems, for 
example hallucinations and delusions, in the pathways to suicidal thoughts and behaviours [9]. In accord with this principle, the CARMS trial aims to examine the extent to which specific experiences of psychosis, such as, the distress caused by hallucinations and delusions are primary triggers for suicidal experiences and/or amplify other known psychological precipitants of suicidal thoughts and experiences, such as, a sense of hopelessness, defeat and entrapment.

The third strength is that the psychological talking therapy, i.e., CBSPp, used in the CARMS trial is suicide focussed, personalised, and formulation driven. This psychological talking therapy is supported by evidence pertaining to acceptability, feasibility and efficaciousness from qualitative and quantitatively designed pilot work with people living in the community with psychosis, prisoners, and psychiatric inpatients $[30,31,43,107-109,121-123]$. These individuals are exceptionally vulnerable to suicidal thoughts, acts and deaths because of their complex, and often severe, mental health problems.

Fourth, CARMS aims to assess not only the efficaciousness of our suicide-focussed psychological intervention, but to advance our understanding of the psychological mechanisms underlying suicidal thoughts and behaviours. It is vitally important to advance these mechanisms in a context of understanding how to address the cyclical, and often, unpredictable patterns in which people transition from, and between suicidal thoughts, urges, plans and acts $[118,119]$.

Fifth, there are four qualitative work streams nested within the CARMS trial. These work streams will probe psychological mechanisms; barriers and facilitators to providing the CARMS trial intervention in the 'realworld'; implementation challenges and solutions; aspects of the therapy which were perceived as being both positive and negative; and the experiences of taking part in suicide research. It has been shown that qualitative work can often contextualise aspects of the design of trials which can remain hidden wherein aspects of interventions may, otherwise, be somewhat tacit [43, 121-126].

Sixth, Patient and Public Involvement (PPI) has been used extensively in the lead-up to this CARMS trial being funded. Furthermore, people who are Experts By Experience with respect to suicidality and severe mental health problems are integral to all stages of the trial which is vital to RCT designs [45].

Four limitations of the CARMS RCT are also worthy of discussion. First, in a meta-analysis of 18 trials of cognitive based therapy for self-harm, the extent to which the therapy was perceived as effective depended on the quality of reporting the effects of the Treatment As Usual (TAU) arm of the trial with a bias in favour of trials which did not provide enough specifics of the TAU arm [127]. In the CARMS RCT an attempt will be made to collect as much information as possible regarding treatment as usual. However, it must be acknowledged that being able to collect this type of information will be challenging for a number of reasons, including, that people may access therapies from a range of sources which may be not be well documented or effectively reported.

Second, the design is single-blinded meaning that neither the therapists nor the participants can be blind to treatment. This is, of course, a necessary limitation. The CARMS researchers carrying out the assessments, those performing data entry in the Clinical Trials Unit, the trial statistician and one Principal Co-Investigator will be blinded to treatment allocation with any unblindings noted. This lends trustworthiness to the data collected and analysed which serves to counter this limitation to an extent.

Third, the participants have experiences of nonaffective psychosis and recent suicidal thoughts and behaviours. That they have recent suicidal experiences is a strength of the CARMS trial. However, a limitation is that it is unclear the extent to which the CARMS therapy, i.e., CBSPp, would need to be adapted for people experiencing other mental health problems together with suicidal experiences. So, it is uncertain whether the therapy used in the CARMS trial is, or can be, generically introduced across mental health services. That said, pilot work with male prisoners and inpatients on psychiatric wards included people with a range of mental health problems, which provides some reassurance that the CARMS CBSPp intervention can be applied to people with a diverse range of mental health problems $[107,108]$.

Fourth, the CARMS trial assesses mechanisms and efficaciousness. It will also examine perceptions of key stakeholders (e.g., service users, mental health service providers and mental health service commissioners) about potential implementation barriers and facilitators within the qualitative work. However, it is limited in assessing whether any benefits of CBSPp can be implemented in mental health services to effect clinical beneficial change in 'real-life' settings, that is, outside of an RCT. That said, participants will be recruited from routine mental health services and the therapists will be individuals seconded from, and/or with experience of, mental health services which off-sets this limitation.

In conclusion, the CARMS RCT addresses a crucially important mental health need which is to determine how to diminish suicidal thoughts, urges, plans and acts in people with severe mental health problems, with a focus on those with non-affective psychosis, who are at elevated risk from death by suicide and who experience severe distress which leads them to have suicidal thoughts, to make 
plans to die by suicide and to make suicide attempts. The CARMS psychological intervention, CBSPp, and the RCT is theoretically grounded and supported by a range of empirical evidence based on numerous research designs in diverse populations.

\section{Abbreviations}

ASIQ: Adult Suicidal Ideation Questionnaire; BPRS-E: Brief Psychiatric Rating Scale; BMJ: British Medical Journal; CARMS: Cognitive AppRoaches to coMbatting Suicidality; CBSP: Cognitive Behavioural Suicide Prevention; CBSPp: Cognitive Behavioural Suicide Prevention for psychosis;

CBT: Cognitive Behavioural Therapy; CDS: Calgary Depression Scale; CONSORT: Consolidated Standards of Reporting Trials; CSRI: Client Service Use Receipt Inventory; CTU: Clinical Trials Unit; DMEC: Data Monitoring and Ethics Committee; DIRUM: Database of Instruments for Resource-use Measurement; EBE: Experts-By-Experience; EME: Efficacy and Mechanism Evaluation; FU: Follow Up; ICD-10: International Statistical Classification of Diseases 10th version; ISRCTN: International Standard Randomised Control Trial Number; ITT: Intention-To-Treat; MAHSC: Manchester Academic Health Sciences Centre; MRC: Medical Research Council; NHS: National Health Service; NIHR: National Institute for Health Research; PANSS: Positive and Negative Syndrome Scale; PPI: Patient and Public Involvement; PSP: Personal and Social Performance Scale; PSS: Personal Social Services; PSYRATS: Psychotic Symptom Rating Scales; QALY: Quality-Adjusted LifeYears; RCT: Randomised Controlled Trial; SAMS: Schematic Appraisal Model of Suicide; SPIRIT: Standard Protocol Items: Recommendations for Intervention Trials; TA: Thematic Analysis; TAU: Treatment As Usual; TIDieR: Template for Intervention Description and Replication; TSC: Trial Steering Committee; UK: United Kingdom; USA: United States of America

\section{Acknowledgements}

We would like to sincerely thank all those individuals who were participants in the CARMS project. We would also like to thank members of our Service User Reference Group (known as the CARMers) who have assisted with numerous and important aspects of CARMS from the inception of the project and include innovative and creative dissemination strategies. Many mental health professionals across NHS trusts and third sector organisation have actively supported and contributed to the CARMS project in ways that are pivotal which we would like to acknowledge.

\section{Trial management}

Trials funded by the MRC are required to be scrutinised by two independent committees. These are the Trial Steering Committee (TSC) and the Data Monitoring and Ethics Committee (DMEC). The MRC provides specific requirements regarding membership of these meetings and the frequency of meetings of each of these committees. The TSC and the DMEC will meet every six months in a co-ordinated fashion, such that the DMEC minutes can feed into the TSC.

\section{Data management}

The data is managed by the Manchester Clinical Trials Unit (CTU). Serious Adverse Events, Adverse Reactions, and Adverse Events will be collated by the CTU according to CARMS protocols and reported to, and assessed by, the independent TSC and DMEC.

\section{Audit}

The CTU, host and sponsor will be responsible for auditing procedures.

\section{Trial sponsor}

University of Manchester UK., Research Governance research Office. Research Governance Practice Manager: Lynne Macrae; Lynne.Macrae@manchester.ac. $u k ;+442,755,436$.

\section{Trial protocol}

Version 4 is the current version. However, recent amendments have been submitted for ethical approval under version 5 .

\section{Disclaimer}

This project is funded by the Efficacy and Mechanism Evaluation (EME)

Programme, an MRC and NIHR partnership (Reference number: 13/161/25).
The views expressed in this publication are those of the author(s) and not necessarily those of the MRC, NIHR or the Department of Health and Social Care. The trial is hosted by Greater Manchester Mental Health NHS Foundation Trust, UK (formerly, Manchester Mental Health and Social Care NHS Trust). The Sponsor is Manchester University, UK.

\section{Authors' contributions}

All authors/co-applicants have read and approved the final manuscript. They will be invited to be co-authors on key academic publications. PAG is the Chief Investigator and Co-Principal investigator. PAG is an expert in the psychology of suicidal experiences and played a central role in all aspects of the CARMS project including, for example, the development of the theoretical mechanisms underpinning the CARMS project, the design of the trial, and patient and public strategies and initiatives. It should be noted that PAG is an Ex;pert-By-Experience in suicidality. PAG was lead author on the current manuscript. DP is a clinical psychologist and played a key role in the development of the suicide-focused psychological therapy used in the CARMS project. DP was also responsible for developing the therapy process measures and, relatedly, the extensive therapist supervision processes and procedures. YA played a central role in developing and leading on Patient and Public Involvement strategies for all stages of the CARMS project from inception to dissemination. In addition, YA had an important role in the design and analysis of the qualitative work streams nested within the CARMS trial. $\mathrm{RD}$ is the CARMS trial psychiatrist. As such, RD was responsible for the development of all psychiatric diagnostic criteria, processes and procedures including those contributing to the eligibility criteria for participation. REll is the CARMS trial health economist and is responsible for the design, execution, and analyses of all health economy aspects of the project. RE is the CARMS trial statistician responsible for all aspects of the analyses including power analyses, analyses of the trial data and moderated mediation analyses of the mechanistic aspects of the CARMS project. CH is the CARMS trial manager. $\mathrm{CH}$ is responsible for numerous central strategic and logistic aspects of the trial. $\mathrm{CH}$ is also central to the examination of therapy process measures. $\mathrm{SJ}$ is a clinical psychologist and experienced triallist in the context of testing the efficaciousness and efficacy of psychological interventions in people with severe mental health problems. This expertise fed into numerous aspects of the CARMS trial design. NK is an epidemiological psychiatrist, an expert in risk factors underlying suicidal thoughts, acts and deaths. NK has significantly shaped clinical practice with respect to suicidality which has had significant influences on mental health policy decision making. These sources of expertise were central to the CARMS project. FL is a clinical psychologist and experienced triallist. FL contributed to central decisions concerning the design of CARMS. In addition FL has considerable expertise in working with people who are Experts-By-Experience in the context of trial design and execution which contributed to Patient and Public Involvement in the CARMS project. $\mathrm{SP}$ is an expert in qualitative methods and analyses across numerous settings in health and clinical psychology. SP has substantial experience of developing qualitative and mixed methods work-streams in the context of clinical and health psychology trials. Hence, SP is the lead for all four qualitative work streams in the CARMS project. GH is a clinical psychologist and CoPrincipal Investigator who has considerable expertise in the design and execution of psychological trials with people with severe mental health problems. GH played a key role in all aspects of the trial design including therapy development, the iterative development and audit of risk assessment protocols, and numerous aspects of Patient and Public Involvement.

\section{Funding}

Funding was obtained from the Efficacy and Mechanism Evaluation (EME) funding stream which is an Medical Research Council, UK, (MRC) and National Institute of Health Research (NIHR) partnership (Reference number: 13/161/25). This funding body had no role in the design of the study, data collection, recruitment strategy, analyses and interpretation apart from requested amendments and clarification required by external peer reviewers and the Board members of the finding body. The funding body has no role in the writing of reports/journal articles arising from the work nor any role in decisions to publish. The sponsor had no role in the design of the study, data collection, recruitment strategy, analyses and interpretation. The sponsor has no role in the writing of reports/journal articles arising from the work nor any role in decisions to publish. 


\section{Availability of data and materials}

The datasets used and/or analysed during the current study are available from the corresponding author on reasonable request.

\section{Ethics approval and consent to participate}

Ethical approval was gained on 11th May 2017 (North West - Greater Manchester South Research Ethics Committee; REC reference: 17/NW/0089; IRAS project ID: 201644). Approval included Participation Information Sheets and Informed Consent forms for participants recruited into the study. All substantial amendments, for example, to the protocol will be submitted for ethical approval.

\section{Consent for publication}

Not Applicable.

\section{Competing interests}

The authors declare that they have no competing interests.

\section{Author details}

'Division of Psychology and Mental Health, School of Health Sciences, Manchester Academic Health Sciences Centre, University of Manchester, Coupland Building 1, Oxford Road, Manchester M13 9PL, UK. ${ }^{2}$ Greater Manchester Mental Health NHS Trust (formerly Manchester Mental Health and Social Care Trust), Manchester, UK. ${ }^{3}$ Manchester Centre for Health Economics, School of Health Sciences, Manchester Academic Health Sciences Centre, University of Manchester, Manchester, UK. ${ }^{4}$ Institute of Psychiatry, Psychology \& Neuroscience, Kings College London, London, UK. ${ }^{5}$ Lancashire Care NHS Foundation Trust, Lancashire, UK. ${ }^{6}$ University of Lancaster, Lancaster, UK.

\section{Received: 21 April 2020 Accepted: 27 May 2020}

Published online: 16 June 2020

\section{References}

1. WHO. Suicide Data 2020 [Available from: https://www.who.int/mental health/prevention/suicide/suicideprevent/en/.

2. AFSP. Suicide Statistics 2020 [Available from: https://afsp.org/about-suicide/ suicide-statistics/.

3. ONS. Suicides in the United Kingdom: 2018 registrations 2019 [Available from: https://www.ons.gov.uk/peoplepopulationandcommunity/ birthsdeathsandmarriages/deaths/bulletins/suicidesintheunitedkingdom/201 8registrations.

4. Samaritans. Suicide Statistics Report 2019 [Available from: https://media. samaritans.org/documents/SamaritansSuicideStatsReport_2019_Dec19_ compressed.pdf.

5. Peterson C, Sussell A, Li J, Schumacher P, Yeoman K, Stone DM. Suicide rates by industry and occupation - National Violent Death Reporting System, 32 states, 2016. MMWR Morb Mortal Wkly Rep. 2020;69(3):57-62.

6. GOV.UK. Serlf harm and suicidal thoughts and attempts 2019 [Available from: https://www.ethnicity-facts-figures.service.gov.uk/health/mentalhealth/adults-reporting-suicidal-thoughts-attempts-and-self-harm/1.5\#datasources.

7. NIMH. Suicide 2019 [Available from: https://www.nimh.nih.gov/health/ statistics/suicide.shtml.

8. Hawton K, van Heeringen K. Suicide. Lancet. 2009:373(9672):1372-81

9. Bolton C, Gooding P, Kapur N, Barrowclough C, Tarrier N. Developing psychological perspectives of suicidal behaviour and risk in people with a diagnosis of schizophrenia: we know they kill themselves but do we understand why? Clin Psychol Rev. 2007;27(4):511-36.

10. Hawton K, Sutton L, Haw C, Sinclair J, Deeks JJ. Schizophrenia and suicide: systematic review of risk factors. Br J Psychiatry. 2005;187:9-20.

11. Saha S, Chant D, McGrath J. A systematic review of mortality in schizophrenia. Is the differential mortality gap worsening over time? Arch Gen Psychiatry. 2007;64(10):1123-31.

12. Cohen S, Lavelle J, Rich CL, Bromet E. Rates and correlates of suicide attempts in first-admission psychotic-patients. Acta Psychiatr Scand. 1994; 90(3):167-71.

13. Palmer BA, Pankratz VS, Bostwick JM. The lifetime risk of suicide in schizophrenia - a reexamination. Arch Gen Psychiatry. 2005;62(3):247-53.
14. Tarrier N, Gooding P, Pratt D, Kelly J, Awenat Y, Maxwell J. Cognitive behavioural prevention of suicide in psychosis: a treatment manual. London: Routledge; 2013.

15. DeVylder JE, Lukens EP, Link BG, Lieberman JA. Suicidal ideation and suicide attempts among adults with psychotic experiences data from the collaborative psychiatric epidemiology surveys. Jama Psychiatry. 2015;72(3):219-25.

16. Chapman CL, Mullin K, Ryan CJ, Kuffel A, Nielssen O, Large MM. Metaanalysis of the association between suicidal ideation and later suicide among patients with either a schizophrenia spectrum psychosis or a mood disorder. Acta Psychiatr Scand. 2015;131(3):162-73.

17. Hawton K, Witt KG, Taylor-Salisbury TL, Arensman E, Gunnell D, Hazell P, et al. Psychosocial interventions for self-harm in adults (review). Cochrane Database Syst Rev. 2016:5:1-308.

18. Meerwijk EL, Parekh A, Oquendo MA, Allen IE, Franck LS, Lee KA. Direct versus indirect psychosocial and behavioural interventions to prevent suicide and suicide attempts: a systematic review and meta-analysis. Lancet Psychiatry. 2016;3(6):544-54.

19. Tarrier N, Taylor K, Gooding P. Cognitive-behavioral interventions to reduce suicide behavior a systematic review and meta-analysis. Behav Modif. 2008; 32(1):77-108.

20. Riblet NBV, Shiner B, Young-Xu Y, Watts BV. Strategies to prevent death by suicide: meta-analysis of randomised controlled trials. Br J Psychiatry. 2017; 210(6):396.

21. Zalsman G, Hawton K, Wasserman D, van Heeringen K, Arensman E, Sarchiapone $M$, et al. Suicide strategies revisited: 10 year systematic review. Lancet Psychiatry. 2016;3(7):649-59.

22. Johnson J, Gooding P, Tarrier N. Suicide risk in schizophrenia: explanatory models and clinical implications, the schematic appraisal model of suicide (SAMS). Psychol Psychother. 2008;81:55-77.

23. Williams JMG. Cry of pain. UK Penguin: Harmondsworth; 1997.

24. Klonsky ED, May AM. The Three-Step Theory (3ST): A new theory of suicide rooted in the "Ideation-to-Action" framework. Int J Cogn Ther. 2015:8(2): 114-29.

25. O'Connor RC. The integrated motivational-volitional model of suicidal behavior. Crisis. 2011;32(6):295-8.

26. Van Orden KA, Witte TK, Cukrowicz KC, Braithwaite SR, Selby EA, Joiner TE. The interpersonal theory of suicide. Psychol Rev. 2010;117(2):575-600.

27. Panagioti M, Tarrier N, Angelakis I, Gooding P. A prospective investigation of the impact of distinct PTSD symptom clusters on suicidal ideation. Cogn Ther Res. 2017;41(4):645-53. https://doi.org/10.1007/s10608-016-9829-2.

28. Taylor PJ, Gooding PA, Wood AM, Johnson J, Pratt D, Tarrier N. Defeat and entrapment in schizophrenia: the relationship with suicidal ideation and positive psychotic symptoms. Psychiatry Res. 2010;178(2):244-8.

29. Panagioti M, Gooding P, Tarrier N. A meta-analysis of the association between Posttraumatic Stress Disorder (PTSD) and suicidality: The role of comorbid depression. Compr Psychiatry. 2012;53(7):915-30.

30. Tarrier N, Kelly J, Maqsood S, Snelson N, Maxwell J, Law H, et al. The cognitive behavioural prevention of suicide in psychosis: a clinical trial. Schizophr Res. 2014;156(2-3):204-10.

31. Pratt D, Tarrier N, Dunn G, Awenat Y, Shaw J, Ulph F, et al. Cognitivebehavioural suicide prevention for male prisoners: a pilot randomized controlled trial. Psychol Med. 2015;45(16):3441-51.

32. Taylor PJ, Gooding P, Wood AM, Tarrier N. The role of defeat and entrapment in depression, anxiety, and suicide. Psychol Bull. 2011;137(3):391-420.

33. Taylor PJ, Gooding PA, Wood AM, Johnson J, Tarrier N. Prospective predictors of suicidality: defeat and entrapment Lead to changes in suicidal deation over time. Suicide Life Threat Behav. 2011:41(3):297-306.

34. Steeg S, Haigh M, Webb RT, Kapur N, Awenat Y, Gooding P, et al. The exacerbating influence of hopelessness on other known risk factors for repeat self-harm and suicide. J Affect Disord. 2016;190(522-528):522-8.

35. Panagioti M, Gooding PA, Tarrier N. A prospective study of suicidal ideation in posttraumatic stress disorder: the role of perceptions of defeat and entrapment. J Clin Psychol. 2015;71(1):50-61.

36. Acosta FJ, Vega D, Torralba L, Navarro S, Ramallo-Fariña Y, Fiuza D, et al. Hopelessness and suicidal risk in bipolar disorder. A study in clinically nonsyndromal patients. Compr Psychiatry. 2012;53(8):1103-9.

37. Beck AT. Hopelessness as a predictor of eventual suicide. Ann N Y Acad Sci. 1986:487:90-6

38. Beck AT, Steer RA, Kovacs M, Garrison B. Hopelessness and eventual suicide - a 10-year prospective-study of patients hospitalized with suicidal ideation. Am J Psychiatr. 1985;142(5):559-63. 
39. Hunter EC, O'Connor RC. Hopelessness and future thinking in parasuicide: the role of perfectionism. Br J Clin Psychol. 2003;42:355-65.

40. Klonsky ED, Kotov R, Bakst S, Rabinowitz J, Bromet EJ. Hopelessness as a predictor of attempted suicide among first admission patients with psychosis: a 10-year cohort study. Suicide Life Threat Behav. 2012;42(1):1-10.

41. O'Connor RC, O'Connor DB, O'Connor SM, Smallwood J, Miles J. Hopelessness, stress, and perfectionism: the moderating effects of future thinking. Cognit Emot. 2004;18(8):1099-120.

42. White RG, McCleery M, Gumley Al, Mulholland C. Hopelessness in schizophrenia: the impact of symptoms and beliefs about illness. J Nerv Ment Dis. 2007;195(12):968-75.

43. Awenat YF, Shaw-Núñez E, Kelly J, Law H, Ahmed S, Welford M, et al. A qualitative analysis of the experiences of people with psychosis of a novel cognitive behavioural therapy targeting suicidality. Psychosis. 2017;9(1):38-47

44. Haddock G, Pratt D, Gooding PA, Peters S, Emsley R, Evans E, et al. Feasibility and acceptability of suicide prevention therapy on acute psychiatric wards: randomised controlled trial. Bjpsych Open. 2019;5(1):e14. https://doi.org/10.1192/bjo.2018.85.

45. Baines R, Donovan J, de Bere SR, Archer J, Jones R. Patient and public involvement in the design, administration and evaluation of patient feedback tools, an example in psychiatry: a systematic review and critical interpretative synthesis. J Health Serv Res Policy. 2019;24(2):130-42.

46. Bodolica V, Spraggon M. Toward patient-centered care and inclusive healthcare governance: a review of patient empowerment in the UAE. Public Health. 2019;169:114-24.

47. Haldane V, Chuah FLH, Srivastava A, Singh SR, Koh GCH, Seng CK, et al. Community participation in health services development, implementation, and evaluation: A systematic review of empowerment, health, community, and process outcomes. PLoS One. 2019;14(5):e0216112. https://doi.org/10. 1371/journal.pone.0216112.

48. Lalani M, Baines R, Bryce M, Marshall M, Mead S, Barasi S, et al. Patient and public involvement in medical performance processes: a systematic review. Health Expect. 2019;22(2):149-61.

49. Walwyn $\mathrm{R}$, Roberts $C$. Therapist variation within randomised trials of psychotherapy: implications for precision, internal and external validity. Stat Methods Med Res. 2010;19:291-315.

50. Batistatou E, Roberts C, Roberts S. Sample size and power calculations for trials and quasi-experimental studies with clustering. Stata J. 2014;14(1):159-75.

51. Reynolds WM. Adult suicidal ideation questionnaire: professional manual. Odessa: Psychological Assessment Resources; 1991.

52. Beck AT, Brown GK, Steer RA, Dahlsgaard KK, Grisham JR. Suicide ideation at its worst point: a predictor of eventual suicide in psychiatric outpatients. Suicide Life Threat Behav. 1999;29(1):1-9.

53. Cull J, Gill W. Suicide probability scale (SPS) manual. Los Angeles: Western Psychological Services; 1988.

54. Beck AT, Steer RA. Manual for Beck scale for suicidal ideation. New York: Psychological Corporation; 1991.

55. Pinninti N, Steer RA, Rissmiller DJ, Nelson S, Beck AT. Use of the Beck scale for suicide ideation with psychiatric inpatients diagnosed with schizophrenia, schizoaffective, or bipolar disorders. Behav Res Ther. 2002; 40(9):1071-9.

56. Gratz KL, Roemer L. Multidimensional assessment of emotion regulation and dysregulation: Development, factor structure, and initial validation of the difficulties in emotion regulation scale. J Psychopathol Behav Assess. 2004; 26:41-54.

57. Hallon LS, Steinman SA, Tolin DF, Diefenbach GJ. Psychometric properties of the difficulties in emotion regulation scale (DERS) and its short forms in adults with emotional disorders. Front Psychol. 2018;9:539.

58. D'Zurilla TJ, Nezu AM. Maydeu-Olivares. Social problem-solving inventoryrevised: technical manual. New York: Multi-Health Systems; 2002.

59. Vaux A, Phillips J, Holly L, Thomson B, Williams D, Stewart D. The social support appraisals (SS-A) scale: studies of reliability and validity. Am J Community Psychol. 1986;14:195-219.

60. Beck AT, Weissman A, Lester D, Trexler $L$. The measurement of pessimism: the hopelessness scale. J Consult Clin Psychol. 1974;42:861-5.

61. Kovacs M, Beck AT, Weissman A. Hopelessness - indicator of suicidal risk. Suicide. 1975;5(2):98-103.

62. Littlewood D, Gooding P, Panagioti M, Kyle S. Nightmares and suicide in posttraumatic stress disorder: the mediating role of defeat, entrapment and hopelessness. J Clin Sleep Med. 2016;12(3):393-9.
63. Panagioti M, Gooding PA, Tarrier N. Hopelessness, defeat, and entrapment in posttraumatic stress disorder their association with suicidal behavior and severity of depression. J Nerv Ment Dis. 2012;200(8):676-83.

64. Gilbert P, Allan S. The role of defeat and entrapment (arrested flight) in depression: an exploration of an evolutionary view. Psychol Med. 1998;28: 585-98.

65. Kay SR, Fiszbein A, Opier LA. The positive and negative syndrome scale (PANSS) for schizophrenia. Schizophr Bull. 1987;13(2):261-76.

66. Haddock G, McCarron J, Tarrier N, Faragher EB. Scale to measure dimensions of hallucinations and delusions: the psychotic symptom rating scales (PSYRATS). Psychol Med. 1999;29:879-89.

67. Morosini PL, Magliano L, Brambilla L. al. e. Development, reliability and acceptability of a new version of the DSM-IV social and occupational functioning assessment scale (SOFAS) to assess routine social functioning. Acta Psychiatr Scand. 2000;101:323-9.

68. Addington D, Addington J, Schissel B. A depression rating scale for schizophrenics. Schizophr Res. 1990:3:247-51.

69. Santor DA, Ascher-Svanum H, Lindenmayer J-P, Obenchain RL. Item response analysis of the positive and negative syndrome scale. BMC Psychiatry. 2007;7(1):66.

70. Bowling A. Research methods in health: Open University press Philadelphia, PA, USA; 2002.

71. Johnston K, Buxton MJ, Jones DR, Fitzpatrick R. Assessing the costs of healthcare technologies in clinical trials. Health Technol Assess. 1999;3(6):1-76.

72. Ridyard $\mathrm{CH}$, Hughes DA. Methods for the collection of resource use data within clinical trials: a systematic review of studies funded by the UK health technology assessment program. Value Health. 2010;13(8):867-72.

73. Beecham J, Knapp M. Costing psychiatric interventions. Costing psychiatric interventions. In: Thornicroft, Graham, ed. Measuring Mental Health Needs (Second Edition). Royal College of Psychiatrists, London. 2001. p. 200-24.

74. Kalra L, Evans A, Perez I, Knapp M, Swift C, Donaldson N. A randomised controlled comparison of alternative strategies in stroke care. Health Technol Assess. 2005;9(18) iii-iv:1-79.

75. Hurley MV, Walsh NE, Mitchell HL, Pimm TJ, Williamson E, Jones RH, et al. Economic evaluation of a rehabilitation program integrating exercise, selfmanagement, and active coping strategies for chronic knee pain. Arthritis Rheum. 2007:57(7):1220-9..

76. Patel A, Maissi E, Chang HC, Rodrigues I, Smith M, Thomas S, et al. Motivational enhancement therapy with and without cognitive behaviour therapy for type 1 diabetes: economic evaluation from a randomized controlled trial. Diabet Med. 2011;28(4):470-9.

77. Robertson W, Stewart-Brown S, Stallard N, Petrou S, Griffiths F, Thorogood $M$, et al. Evaluation of the effectiveness and cost-effectiveness of families for health V2 for the treatment of childhood obesity: study protocol for a randomized controlled trial. Trials. 2013;14:81.

78. Barton GR, Hodgekins J, Mugford M, Jones PB, Croudace T, Fowler D. Measuring the benefits of treatment for psychosis: validity and responsiveness of the EQ-5D. Br J Psychiatry. 2009;195(2):170-7.

79. Saha S, Bejerholm U, Gerdtham UG, Jarl J. Cost-effectiveness of supported employment adapted for people with affective disorders. Nordic J Psychiatry. 2018:(3):236-9. https://doi.org/10.1080/08039488.2017.

80. Knapp M, Windmeijer F, Brown J, Kontodimas S, Tzivelekis S, Haro JM, et al. Cost-utility analysis of treatment with olanzapine compared with other antipsychotic treatments in patients with schizophrenia in the panEuropean SOHO study. Pharmacoeconomics. 2008;26(4):341-58.

81. Mulhern B, Mukuria C, Barkham M, Knapp M, Byford S, Soeteman D, et al. Using generic preference-based measures in mental health: psychometric validity of the EQ-5D and SF-6D. Br J Psychiatry. 2014;205(3):236-43.

82. Devlin NJ, Shah KK, Feng Y, Mulhern B, van Hout B. Valuing health-related quality of life: an EQ-5D-5L value set for England. Health Econ. 2018;27(1):7-22.

83. Feng $Y$, Devlin N, Herdman M. Assessing the health of the general population in England: how do the three- and five-level versions of EQ-5D compare? Health Qual Life Outcomes. 2015;13(1):1-16.

84. Janssen MF, Pickard AS, Golicki D, Gudex C, Niewada M, Scalone L, et al. Measurement properties of the EQ-5D-5L compared to the EQ-5D-3L across eight patient groups: a multi-country study. Qual Life Res. 2013;22(7):1717-27.

85. Golicki D, Niewada M, Buczek J, Karlińska A, Kobayashi A, Janssen MF, et al. Validity of EQ-5D-5L in stroke. Qual Life Res. 2014;24(4):845-50.

86. Golicki D, Niewada M, Karlinska A, Buczek J, Kobayashi A, Janssen MF, et al. Comparing responsiveness of the EQ-5D-5L, EQ-5D-3L and EQ VAS in stroke patients. Qual Life Res. 2015;24(6):1555-63. 
87. Mulhern B, Feng Y, Shah K, Janssen MF, Herdman M, van Hout B, et al. Comparing the UK EQ-5D-3L and English EQ-5D-5L value sets. Pharmacoeconomics. 2018;36(6):699-713.

88. Horvath AO, Greenberg LS. Development and validation of the working Alliance inventory. J Couns Psychol. 1989;36(2):223-33.

89. Baron RM, Kenny DA. The moderator-mediator variable distinction in social psychological research: Conceptual, strategic, and statistical considerations. J Pers Soc Psychol. 1986;51(6):1173-82.

90. Dunn G, Fowler D, Rollinson R, Freeman D, Kuipers E, Smith B, et al. Effective elements of cognitive behaviour therapy for psychosis: results of a novel type of subgroup analysis based on principal stratification. Psychol Med. 2012;42(5):1057-68.

91. Dunn G. Pragmatic trials of complex interventions: methodological challenges. Epidemiol Psychiatr Sci. 2013;22:105-9.

92. Emsley RA, Dunn G, White IR. Mediation and moderation of treatment effects in randomised controlled trials of complex interventions. Stat Methods Med Res. 2010;19(3):237-70.

93. Johnson J, Wood AM, Gooding P, Taylor PJ, Tarrier N. Resilience to suicidality: the buffering hypothesis. Clin Psychol Rev. 2011;31(4):563-91.

94. Braun V, Clarke V. Using thematic analysis in psychology. Qual Res Psychol. 2006;3(2):77-101.

95. Boyatzis RE. Transforming qualitative information. Cleveland: Sage; 1998.

96. Henwood K, Pidgeon N. Qualitative research and psychological theorizing. Br J Psychol. 1992;83(1):97-111.

97. Peters S. Qualitative research methods in mental health. Evid Based Menta Health. 2010;13:35-40.

98. Beecham J, Knapp M. Costing psychiatric interventions. Measuring mental health needs. Measuring mental health needs; 2000. p. 200-24.

99. Kalra L, Evans A, Perez I, Knapp M, Swift C, Donaldson N. A randomised controlled comparison of alternative strategies in stroke care. Health Technol Assess. 2005;9(18):1-79.

100. Robertson W, Stewart-Brown S, Stallard N, Petrou S, Griffiths F, Thorogood $M$, et al. Evaluation of the effectiveness and cost-effectiveness of families for health V2 for the treatment of childhood obesity: study protocol for a randomized controlled trial. Trials. 2013;14(1):81.

101. Personal Social Services Research Unit P. Unit Costs of Health and Social Care 2016.

102. Do H. NHS reference costs 2015 to 2016. London: Department of Health; 2016.

103. Hansson C, Joas E, Palsson E, Hawton K, Runeson B, Landen M. Risk factors for suicide in bipolar disorder: a cohort study of 12850 patients. Acta Psychiatr Scand. 2018;138(5):456-63.

104. Bang M, Park JY, Kim KR, Lee SY, Song YY, Kang Jl, et al. Suicidal ideation in individuals at ultra-high risk for psychosis and its association with suspiciousness independent of depression. Early Interv Psychiatry. 2019; 13(3):539-45.

105. Chouinard VA, Shinn AK, Valeri L, Chouinard PA, Gardner ME, Asan AE, et al. Visual hallucinations associated with multimodal hallucinations, suicide attempts and morbidity of illness in psychotic disorders. Schizophr Res. 2019;208:196-201.

106. Simon GE, Yarborough BJ, Rossom RC, Lawrence JM, Lynch FL, Waitzfelder $\mathrm{BE}$, et al. Self-reported suicidal ideation as a predictor of suicidal behavior among outpatients with diagnoses of psychotic disorders. Psychiatr Serv. 2019;70(3):176-83

107. Haddock G, Pratt D, Gooding P, Peters S, Emsley R, Evans E, et al. Feasibility and acceptability of suicide prevention therapy on acute psychiatric wards: randomised controlled trial. Br J Psychiatry Open. 2019;5(1):e14.

108. Pratt D, Gooding P, Eccles S, Awenat $Y$, Tarrier N. Cognitive Behavioural suicide prevention for male prisoners: case examples. Cogn Behav Pract. 2016;23(4):485-501.

109. Pratt D, Gooding P, Kelly J, Johnson J, Tarrier N. Case formulation in suicidal behaviour. In: Tarrier $\mathrm{N}$, editor. Case formulation in cognitive behaviour therapy: the treatment of challenging and complex cases. London: Routledge; 2015

110. Johnson J, Tarrier N, Gooding P. An investigation of aspects of the cry of pain model of suicide risk: the role of defeat in impairing memory. Behav Res Ther. 2008;46(8):968-75.

111. Littlewood D, Gooding P, Panagioti M, Kyle S. Investigating psychological mechanisms in relation to sleep problems and suicide. J Clin Sleep Med. 2016;12(6):931.

112. Owen R, Dempsey R, Jones S, Gooding P. Defeat and entrapment in bipolar disorder: exploring the relationship with suicidal ideation from a psychological theoretical perspective. Suicide Life Threat Behav. 2018;48(1): 116-28.

113. Owen R, Gooding P, Dempsey R, Jones S. A qualitative investigation into the relationships between social factors and suicidal thoughts and acts experienced by people with a bipolar disorder diagnosis. J Affect Disord. 2015;176:133-40.

114. Palmier-Claus JE, Taylor PJ, Gooding P, Dunn G, Lewis SW. Affective variability predicts suicidal ideation in individuals at ultra-high risk of developing psychosis: an experience sampling study. Br J Clin Psychol. 2012 51(1):72-83

115. Panagioti M, Gooding P, Taylor PJ, Tarrier N. Negative Self-Appraisals and Suicidal Behavior Among Trauma Victims Experiencing PTSD Symptoms: The Mediating Role of Defeat and Entrapment. Depress Anxiety. 2012;29(3): 187-94.

116. Pratt D, Gooding P, Johnson J, Taylor P, Tarrier N. Suicide schemas in nonaffective psychosis: an empirical investigation. Behav Res Ther. 2010;48(12): 1211-20.

117. Taylor PJ, Wood AM, Gooding P, Tarrier N. Appraisals and suicidality: the mediating role of defeat and entrapment. Arch Suicide Res. 2010;14(3):236-47.

118. O'Connor RC, Nock MK. The psychology of suicidal behaviour. Lancet Psychiatry. 2014;1(1):73-85.

119. O'Connor RC, Portzky G. Looking to the future: a synthesis of new developments and challenges in suicide research and prevention. Front Psychol. 2018:9:2139.

120. Panagioti M, Gooding P, Taylor PJ, Tarrier N. A model of suicidal behavior in posttraumatic stress disorder (PTSD): the mediating role of defeat and entrapment. Psychiatry Res. 2013;209(1):55-9.

121. Awenat Y, Peters S, Shaw-Nunez E, Gooding P, Pratt D, Haddock G. Staff experiences and perceptions of working with in-patients who are suicidal: qualitataive analysis. Br J Psychiatry. 2017;211(2):103-8.

122. Awenat YF, Moore C, Gooding PA, Ulph F, Mirza A, Pratt D. Improving the quality of prison research: a qualitative study of ex-offender service user involvement in prison suicide prevention research. Health Expect. 2018; 21(1):100-9.

123. Awenat YF, Peters S, Gooding PA, Pratt D, Shaw-Nunez E, Harris K, et al. A qualitative analysis of suicidal psychiatric inpatients views and expectations of psychological therapy to counter suicidal thoughts, acts and deaths. Bmc Psychiatry. 2018;18:334

124. Berry K, Haddock G, Kellett S, Awenat Y, Szpak K, Barrowclough C. Understanding outcomes in a randomized controlled trial of a Ward-based intervention on psychiatric inpatient wards: a qualitative analysis of staff and patient experiences. J Clin Psychol. 2017;73(10):1211-25.

125. Bucci S, Morris R, Barrowclough C, Berry N, Haddock G, Lewis S, et al. Staff and Service User Perspectives of Digital Technology for Early Psychosis. Early Interven Psychiatry. 2018;12:31.

126. Le Geyt G, Awenat Y, Tai S, Haddock G. Personal accounts of discontinuing neuroleptic medication for psychosis. Qual Health Res. 2017;27(4):559-72.

127. Witt K, de Moraes DP, Salisbury TT, Arensman E, Gunnell D, Hazell P, et al. Treatment as usual (TAU) as a control condition in trials of cognitive behavioural-based psychotherapy for self-harm: impact of content and quality on outcomes in a systematic review. J Affect Disord. 2018;235:434-47.

\section{Publisher's Note}

Springer Nature remains neutral with regard to jurisdictional claims in published maps and institutional affiliations.

Ready to submit your research? Choose BMC and benefit from:

- fast, convenient online submission

- thorough peer review by experienced researchers in your field

- rapid publication on acceptance

- support for research data, including large and complex data types

- gold Open Access which fosters wider collaboration and increased citations

- maximum visibility for your research: over $100 \mathrm{M}$ website views per year

At $\mathrm{BMC}$, research is always in progress.

Learn more biomedcentral.com/submission 